\title{
EVALUATION OF BEARING CAPACITY OF STRIP FOOTING USING RANDOM LAYERS CONCEPT
}

\author{
MAREK KAWA, DARIUSZ ŁYDŻBA \\ Wrocław University of Technology, Wrocław, Poland. E-mail: marek.kawa@pwr.edu.pl
}

\begin{abstract}
The paper deals with evaluation of bearing capacity of strip foundation on random purely cohesive soil. The approach proposed combines random field theory in the form of random layers with classical limit analysis and Monte Carlo simulation. For given realization of random the bearing capacity of strip footing is evaluated by employing the kinematic approach of yield design theory. The results in the form of histograms for both bearing capacity of footing as well as optimal depth of failure mechanism are obtained for different thickness of random layers. For zero and infinite thickness of random layer the values of depth of failure mechanism as well as bearing capacity assessment are derived in a closed form. Finally based on a sequence of Monte Carlo simulations the bearing capacity of strip footing corresponding to a certain probability of failure is estimated. While the mean value of the foundation bearing capacity increases with the thickness of the random layers, the ultimate load corresponding to a certain probability of failure appears to be a decreasing function of random layers thickness.
\end{abstract}

Key words: random field theory, reliability, bearing capacity, random layers concept, kinematic approach

\section{INTRODUCTION}

Soil is a natural medium with properties being a result of complex and long-term weathering and sedimentation processes. The consequence of this obvious fact is a strong spatial variability of mechanical and physical properties of the soil medium. While, the soil is commonly used for supporting constructions, variability of its properties may have a significant impact on the safety of the designed foundations or slopes. Developing methods for probabilistic modeling of geotechnical problems with regard to the variability and randomness of the soil properties is a subject of interest for many researchers.

Recently in the probabilistic modeling of soil medium the methods that combine Monte-Carlo simulation with Random Fields Theory seem to be of particular interest. The most popular among these methods is probably Random Finite Element Method (Griffiths and Fenton 2001, Fenton and Griffiths 2003) which uses classical FEM analysis in order to solve individual Monte-Carlo realization. Since the method is very time-consuming in order to improve its efficiency some combined approaches has been developed. To mention just two examples Al-Bittar and Sobura (2012) have used sparse polynomial chaos expansion in order to reduce number of FEM calculations for probabilistic analysis and Kasama et. al. (2012) proposed efficient approach in which classical formulation of FEM has been replaced with FE formulation of limit analysis.

It is evident that obtaining accurate results with the methods, which bases on the random field theory depends mostly on the generation of the random fields, which satisfactorily reconstruct the statistical measures of real fields of soil properties. As has been stated in some recent works (Vessia et al. 2009, Pieczyńska-Kozłowska et. al. 2015) generating isotropic field for that purpose can result in inaccurate results and should not be accepted in designing practice. The so-called fluctuation scale, in the case of soil medium is usually several times greater in the horizontal than in the vertical direction. This means that the parameters of the soil change along the horizontal direction far less rapidly than along the vertical one. Taking that into account it seems that when same simplification of the random field is to be used the assumption that the soil medium consist of horizontal layers with finite thickness and infinite length (horizontal scale of fluctuation equal to infinity) should be associated with relatively small error.

In the present work the concept of random layers has been applied for assessment of bearing capacity of the strip footing within the framework of reliability theory. The evaluation is obtained as an optimized 
solution of kinematic failure mechanism. The applied method allows for probability analysis of both estimated value of bearing capacity as well as the depth of failure mechanism. For zero and infinite thickness of random layer the values of depth of failure mechanism as well as bearing capacity assessment has been obtained analytically. In the final part of the work, the loads corresponding to a certain probability of foundation failure, has been determined.

\section{FORMULATION OF THE PROBLEM}

Spatial variability of soil parameters in random field along specified direction can be conveniently measured by value of so-called fluctuation scale (Vanmarcke 1983), i.e., the length within which the points of random field are significantly correlated. According to the results of in-situ tests (presented for instance in wok by Uzielli et. al. 2006) the horizontal scale of fluctuation is usually 10 to 100 times greater than in the vertical direction. That means that the changes in soil properties along horizontal direction are 10 to 100 times less rapid than the changes in properties along vertical direction. In the view of that fact it could be assumed that the scale of fluctuation in horizontal direction (in comparison with vertical direction) is equal to infinity. As the result of this assumption the random field with values changing only in vertical direction can be approximated by the random layers. The assumption that the parameters of the soil medium can be satisfactorily represented by set of horizontal random layers is a crucial for this study.
The generated random field is always one of the infinite number of possible realizations, therefore for probabilistic analysis the random field generators are usually used in the framework of Monte-Carlo simulation. To solve the boundary value problem for individual realization the Finite Element or Finite Difference Method are usually used. Since calculations performed by such methods usually takes a lot of time, the whole procedure is a very time-consuming one, especially for non-linear problems such us determining of critical load for elastic-plastic medium. Alternative, less time-consuming approach could be to assess the bearing capacity of the soil medium using limit state theorems. Such an approach is utilized in this paper

Exemplary field of random layers together with assumed kinematic failure mechanism and hodograph of velocities is shown in Fig. 1. Soil is assumed as weightless and purely cohesive, so cohesion is the only random variable in the problem. All the blocks of mechanism are assumed to be rigid. Additionally, it is assumed that the horizontal discontinuity line $l_{2}$ may lie only along contact of two subsequent computational layers. Thus, estimation of load capacity is made only for specified depths of failure mechanism $h$ equal to multiples of a thickness of the computational layer.

For the presented failure mechanism the rate of plastic dissipation is expressed as:

$$
\dot{D}=2\left(\left(v_{12} l_{12}+v_{23} l_{23}+v_{3} l_{3}\right) c_{1}+v_{2} l_{2} c_{2}\right)
$$

where distances $l_{i}$ and velocities $v_{i}$ are show in Fig. 1, $c_{1}$ denotes average cohesion, identical along lines $l_{12}$, $l_{23}, l_{3}$ and $c_{2}$ denotes the cohesion along line $l_{2}$. Due to

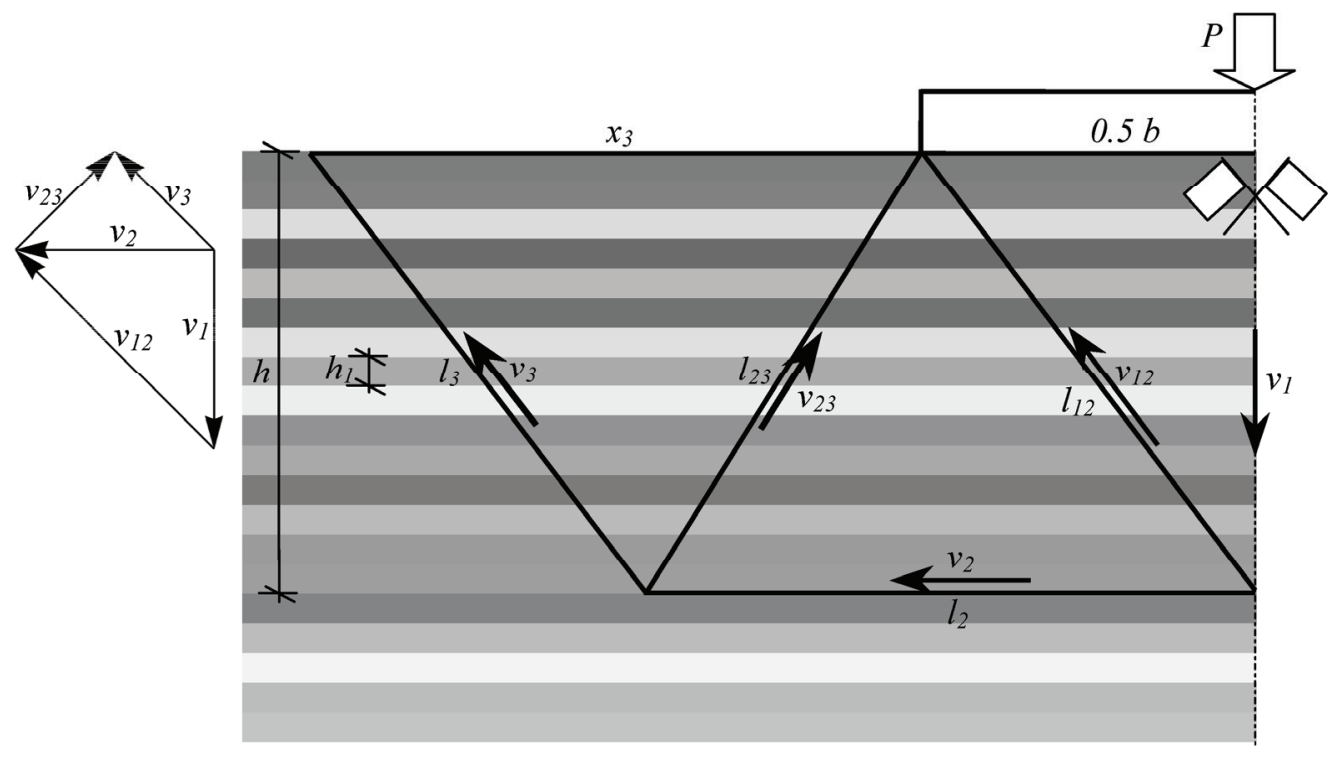

Fig. 1. The assumed failure mechanism with hodograph of velocities 
the assumption that considered soil is weightless the upper bound estimation of bearing capacity of footing is actually equal to formula (1) divided by $v_{1}$.

It is worth noting. that knowing width of footing $b$ and depth of failure mechanism $h$, all other lengths can be expressed as the function of these values and, for instance, $l_{2}$ and $x_{3}$ (Fig. 1) as:

$$
\begin{gathered}
l_{12}=\sqrt{\left(\frac{1}{2} b\right)^{2}+h^{2}}, \quad l_{23}=\sqrt{\left(l_{2}-\frac{1}{2} b\right)^{2}+h^{2}}, \\
l_{3}=\sqrt{\left(x_{3}+\frac{1}{2} b-l_{2}\right)^{2}+h^{2}}
\end{gathered}
$$

Also all the velocities can be expressed as the function of $v_{1}$ and the respective lengths as:

$$
\begin{gathered}
v_{2}=\frac{b}{2 h} v_{1}, \quad v_{12}=\frac{l_{12}}{h} v_{1}, \\
v_{3}=\frac{l_{3}}{x_{3}} \frac{b}{2 h} v_{1}, \quad v_{23}=\frac{l_{23}}{x_{3}} \frac{b}{2 h} v_{1} .
\end{gathered}
$$

As can be seen in Fig. $1 c_{1}$ being cohesion along the lines $l_{12}, l_{23}$ and $l_{3}$ is equal to average over all layers located in the range of the failure mechanism, and $c_{2}$ is equal to minimal cohesion of two layers contacting along line $l_{2}$. For specified realization and given depth of failure mechanism $h$ both of these values can be easily calculated. Knowing $c_{1}$ and $c_{2}$ the geometry of the mechanism can be optimized. By substituting (2) and (3) into (1), differentiating with respect to $l_{2}$ and $x_{3}$ and equating derivatives to 0 optimal values of $l_{2}$ and $x_{3}$ are obtained as:

$$
\begin{gathered}
l_{2}=\frac{b}{2}-\frac{h\left(c_{2}-2 c_{1}\right)}{\sqrt{4 c_{1}^{2}+4 c_{1} c_{2}-c_{2}^{2}}}, \\
x_{3}=\frac{4 c_{1} h}{\sqrt{4 c_{1}^{2}+4 c_{1} c_{2}-c_{2}^{2}}} .
\end{gathered}
$$

Substituting (2)-(4) into (1) and dividing by $v_{1}$ allow expressing the final form of upper bound assessment of bearing capacity for considered mechanism as:

$$
P \leq \frac{b^{2}\left(c_{1}+c_{2}\right)+2 b \sqrt{4 c_{1}^{2}+4 c_{1} c_{2}-c_{2}^{2}}+4 c_{1} h^{2}}{2 h} .
$$

Monte-Carlo procedure can be now carried out as follows: For any single realization values of cohesion are randomly assigned to individual layers in accordance with adopted vertical correlation function for the field. Then, assuming depths of mechanism of failure $h$ equal to subsequent multiples of the thickness of computational layer the values of $c_{1}$ and $c_{2}$ are determined and the value of the assessment (5) is calculated. The final value of the bearing capacity assessment for particular realization is equal to a minimum of values obtained for all the considered depths. This minimum also indicates which of all the values of $h$ is an optimal one. All steps are then repeated for the subsequent realization.

The proposed method is very efficient. The numerical procedure written in the Mathematica package on the modern PC takes 3.5 to 22 minutes to obtain results for 10 to 200 layers field and 100000 Monte Carlo realizations. The results allow for the random analysis of both the assessment of bearing capacity of the footing as well as the optimal depth of failure mechanism. Although the results are obtained in a discrete manner only for mechanisms with depth $h$ equal to multiples of the assumed thickness of layer, the precision of obtaining exact value of $h$ can be easily improved by increasing the density of computational discretization of the field (the random layers can be also divided into a few additional computational layers).

The presented numerical procedure allows for the adoption of any correlation structure in the vertical direction. In this paper it has been assumed that cohesion, which is the only random variable, is assigned for each layer independently. The autocorrelation function of the random field corresponding to random layers realization defined as:

$$
R(\tau)=\frac{E\left[\left(c(x)-\mu_{c}\right)\left(c(x+\tau)-\mu_{c}\right)\right]}{\sigma_{c}^{2}}
$$

is presented in Fig. 2.

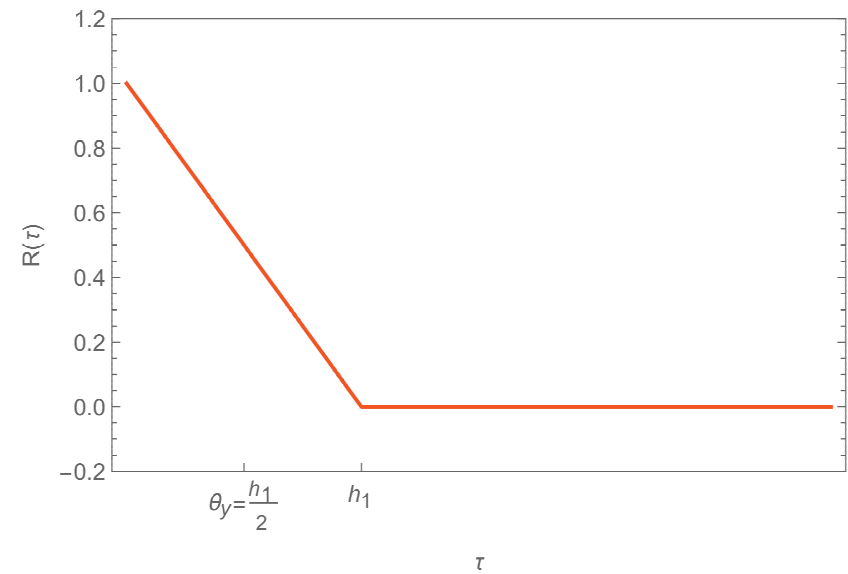

Fig. 2. Autocorrelation function and fluctuation scale for assumed field structure 
The operator $E[$.$] denotes expectation value, c(x)$ is the function of cohesion and $\mu_{c}$ and $\sigma_{c}^{2}$ are mean and variance of cohesion respectively. As can be seen the function descends linearly from one (meaning the full correlation) to zero (meaning no correlation) for length $\tau$ from zero to thickness of random layer $h_{1}$. For all values of $\tau$ grater then $h_{1}$ the value of the function is zero. Classically the correlation with value greater than 0.5 is a interpreted as significant. Therefore, for the assumed field structure the scale of fluctuation is equal to a half of the thickness of random layer.

\section{NUMERICAL RESULTS}

Simulation has been performed for two cases: a small and a large range of cohesion variation in the field. In both cases, the cohesion has been described with uniform probability distribution: the range of variation has been assumed from $10 \mathrm{kPa}$ to $20 \mathrm{kPa}$ and from $10 \mathrm{kPa}$ to $40 \mathrm{kPa}$, respectively. For both of these cases three different thicknesses of the random layers have been considered, namely: $0.5 \mathrm{~m}, 0.05 \mathrm{~m}$ and $0.01 \mathrm{~m}$. In all the simulations carried out it has been assumed that the width of the foundation is equal to $1 \mathrm{~m}$ and the depth of the domain is $2 \mathrm{~m}$ (depth of failure mechanism has been always equal or less than that). For each considered case Monte-Carlo simulation consisted on 100000 realizations.

The results in the form of histograms and estimated normal distributions of both assessment of bearing capacity $P$ as well as the range of the failure mechanism $h$ have been shown in Figs. 3-8. The mean and standard deviation have been estimated using the formulas:

$$
\begin{gathered}
\mu_{J} \approx \frac{1}{N} \sum_{i=1}^{N} J_{i}, \quad \sigma_{J}=\sqrt{\sigma_{J}^{2}}=\frac{1}{N-1} \sum_{i=1}^{N}\left(J_{i}-\mu_{J}\right)^{2}, \\
\text { where } J \in\{P, h\}
\end{gathered}
$$

and are collected in Table 1. As can be seen in both: the figures and the table the mean of bearing capacity assessment is grater for greater mean value of cohesion, which is obvious. On the contrary, the range of failure mechanism seems to be slightly smaller for grater mean value of cohesion. For both assumed
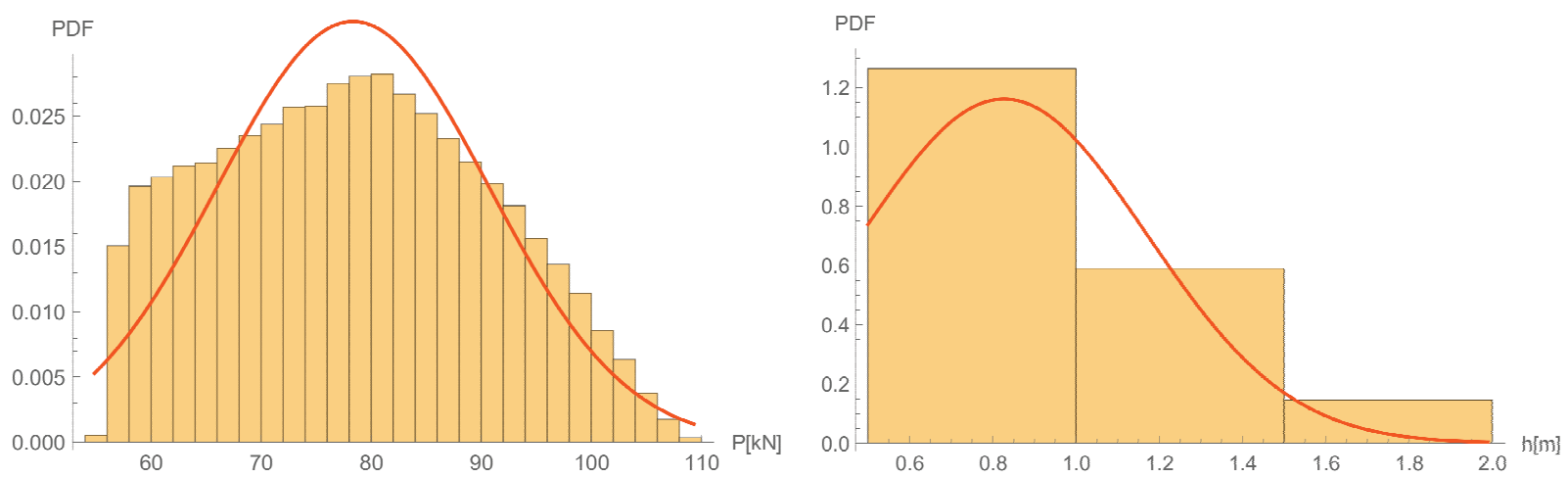

Fig. 3. Histogram and estimated normal distribution. Left: assessment of bearing capacity; right: depth of failure mechanism. Cohesion range 10-20 kPa, thickness of random layer $0.5 \mathrm{~m}$
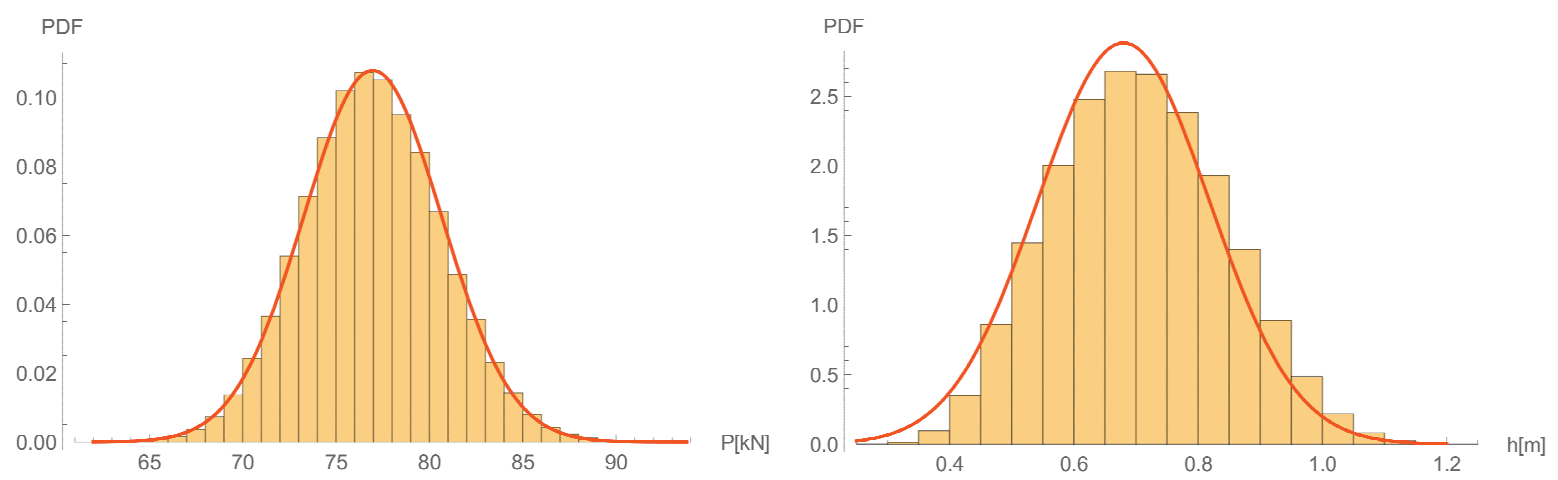

Fig. 4. Histogram and estimated normal distribution. Left: assessment of bearing capacity; right: depth of failure mechanism. Cohesion range $10-20 \mathrm{kPa}$, thickness of random layer $0.05 \mathrm{~m}$ 

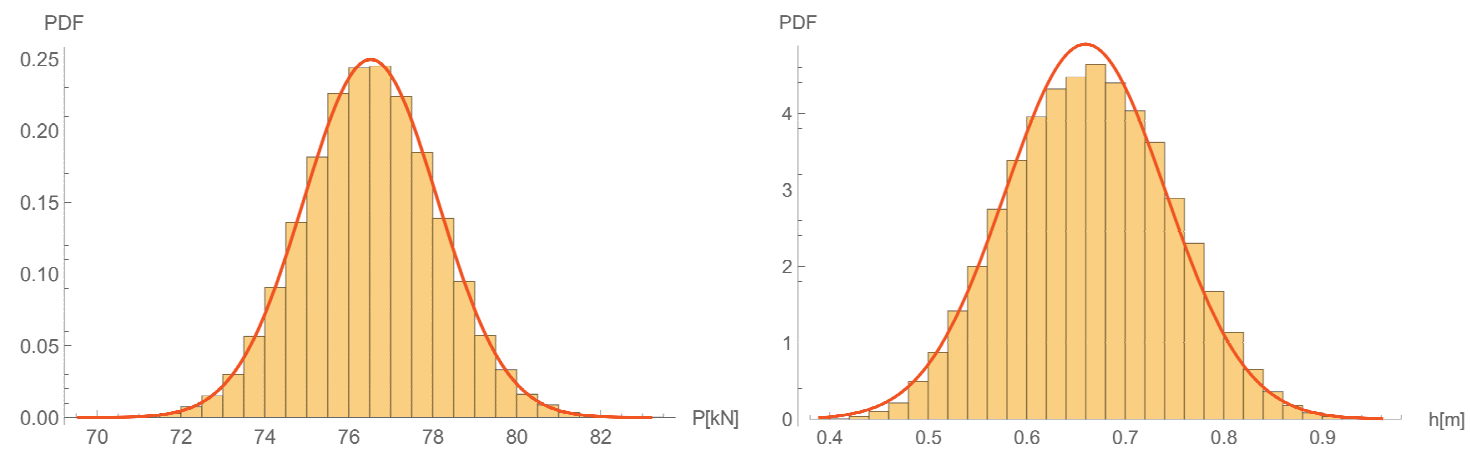

Fig. 5. Histogram and estimated normal distribution. Left: assessment of bearing capacity; right: depth of failure mechanism. Cohesion range 10-20 kPa, thickness of random layer $0.01 \mathrm{~m}$
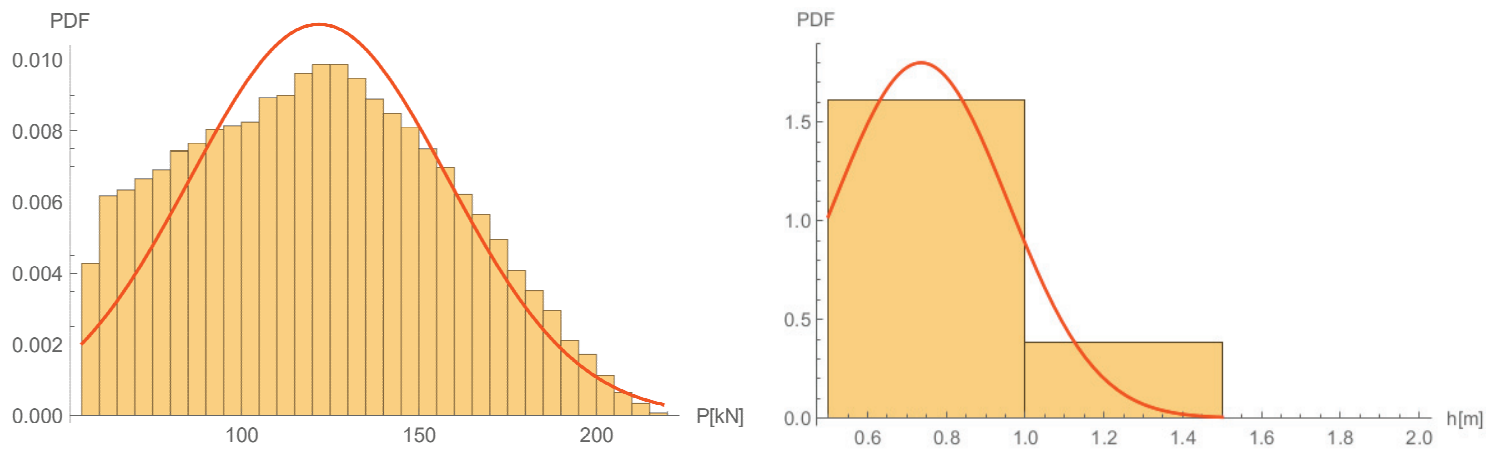

Fig. 6 Histogram and estimated normal distribution. Left: assessment of bearing capacity; right: depth of failure mechanism. Cohesion range $10-100 \mathrm{kPa}$, thickness of random layer $0.5 \mathrm{~m}$
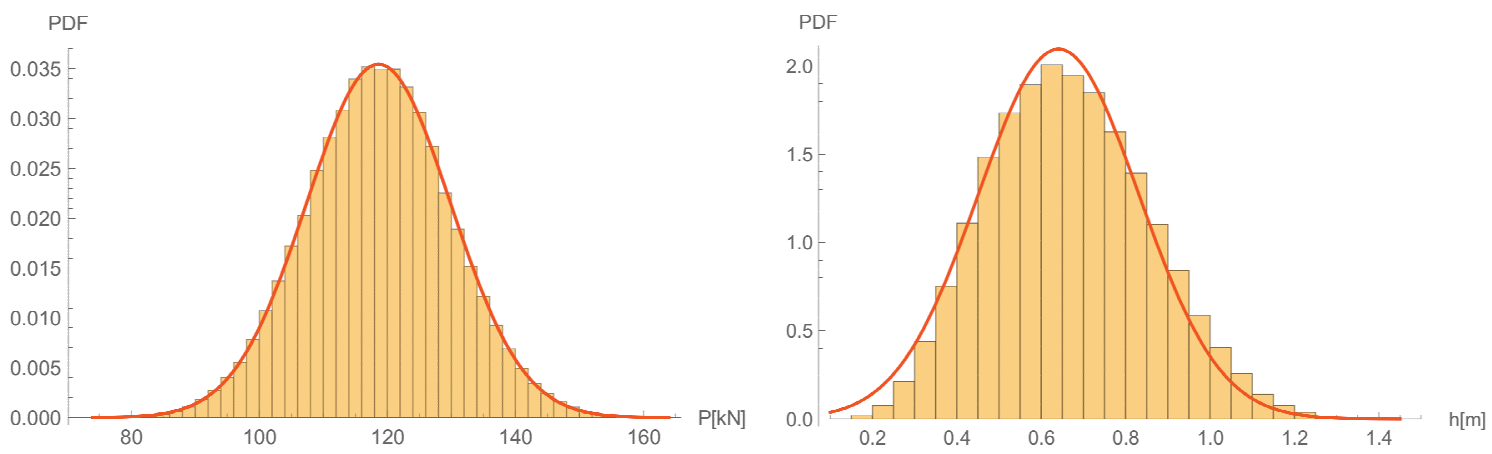

Fig. 7. Histogram and estimated normal distribution. Left: assessment of bearing capacity; right: depth of failure mechanism. Cohesion range $10-100 \mathrm{kPa}$, thickness of random layer $0.05 \mathrm{~m}$
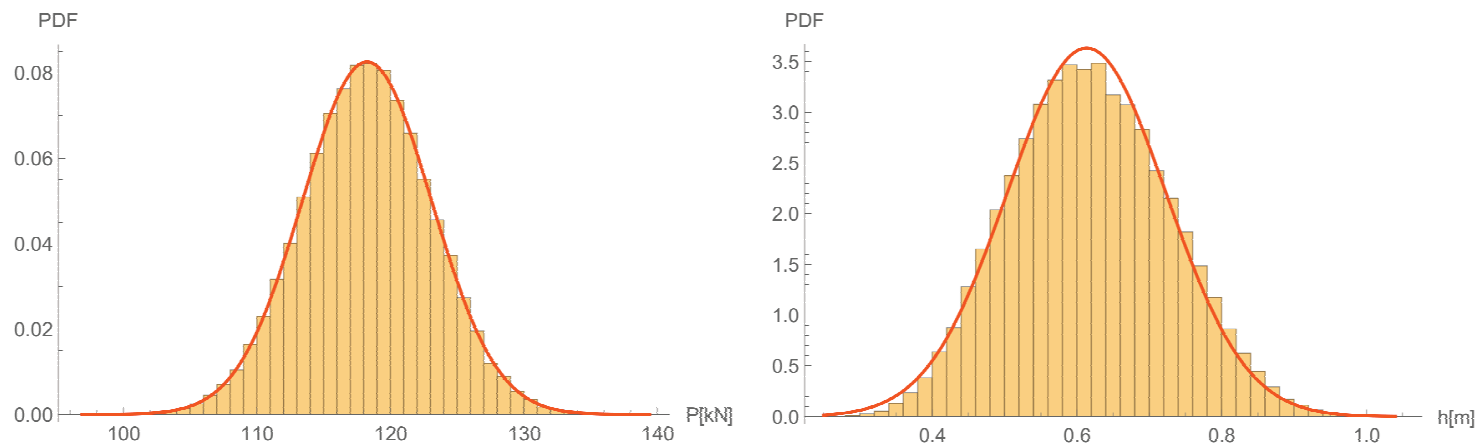

Fig. 8. Histogram and estimated normal distribution. Left: assessment of bearing capacity; right: depth of failure mechanism. Cohesion range $10-100 \mathrm{kPa}$, thickness of random layer $0.01 \mathrm{~m}$ 
Table 1. Parameters of estimated normal distribution for the result obtained in simulations

\begin{tabular}{|c|c|c|c|c|c|c|c|c|c|}
\hline \multicolumn{2}{|c|}{ Range of cohesion [kPa] } & \multicolumn{4}{c|}{$10-20$} & \multicolumn{4}{c|}{$10-40$} \\
\hline \multicolumn{2}{|c|}{ Quantity } & \multicolumn{2}{c|}{$P[\mathrm{kN}]$} & \multicolumn{2}{c|}{$h[\mathrm{~m}]$} & \multicolumn{2}{c|}{$P[\mathrm{kN}]$} & \multicolumn{2}{c|}{$h[\mathrm{~m}]$} \\
\hline \multicolumn{2}{|c|}{ Mean and stanard deviation } & $\mu_{P}$ & $\sigma_{P}$ & $\mu_{h}$ & $\sigma_{h}$ & $\mu_{P}$ & $\sigma_{P}$ & $\mu_{h}$ & $\sigma_{h}$ \\
\hline \multirow{2}{*}{$\begin{array}{l}\text { Random layer } \\
\text { thickness [m] }\end{array}$} & 0.01 & 76.5 & 1.59 & 0.660 & 0.082 & 118.2 & 4.83 & 0.613 & 0.110 \\
\cline { 2 - 11 } & 0.05 & 77.0 & 3.72 & 0.680 & 0.139 & 118.6 & 11.25 & 0.641 & 0.190 \\
\hline
\end{tabular}

variability ranges, it can be observed that with the decreasing value of thickness of random layer the standard deviation of both bearing capacity assessment $P$ and mechanism depth $h$ also decreasing. Such decreasing can be also observed in mean value of $P$ for both assumed ranges of cohesion variation.

Let us assume that thickness of random layer tends to zero. Since $c_{1}$ is the average value of cohesion of all the layers located within the depth of failure mechanism, in the considered case it is equal to the average of an infinite number of layers, i.e. to the mean value of cohesion in the field. Additionally, in any layer with finite thickness grater then zero which contains line $l_{2}$ also the infinite number of random layers with values of cohesion from the whole range of cohesion variation are located. The value of $c_{2}$ is equal to one of these values and the optimal value of assessment is obtained when it is a value as small as possible. This means that the best upper bound of bearing capacity of the footing is achieved when the line $l_{2}$ appears in layer with cohesion equal to the lowest value of cohesion variation. Since values of $c_{1}$ and $c_{2}$ do not depend on the realization and are constant in the field, both depth of failure mechanism and assessment of bearing capacity of the footing can be determined analytically. Differentiating expression (5) with respect to $h$ and equating derivatives to zero the optimum $h$ is obtained as:

$$
h=\frac{b}{2} \sqrt{\frac{c_{1}+c_{2}}{c_{1}}} .
$$

Subsequently substituting (8) into (5) assessment of the force $P$ is determined as:

$$
P \leq b\left(2 \sqrt{c_{1}} \sqrt{c_{1}+c_{2}}+\sqrt{4 c_{1}^{2}+4 c_{1} c_{2}-c_{2}^{2}}\right) .
$$

The variation for both of these values is equal to zero.

The formulas (8) and (9) can be also useful in the case of infinite thickness of random layer. For that case, the mechanism is actually located in a single layer with the cohesion depending only on realization. This means that in all realization $c_{1}$ and $c_{2}$ are equal to each other. Hence (8) becomes:

$$
h=\frac{b}{2} \sqrt{2}
$$

and (9) simplifies to:

$$
P \leq b(2 \sqrt{2}+\sqrt{7}) c
$$

The depth of failure mechanism (10) for that case does not depend on the realization and thus its variation is equal to zero. Contrary the assessment of bearing capacity (11) depends on the realization but since the formula is a linear one it easy to conclude that

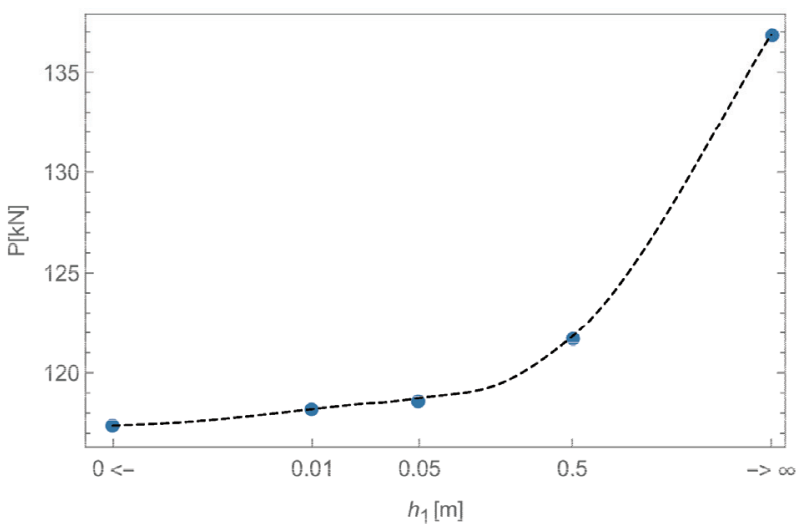

Fig. 9. Mean value of assessment of bearing capacity for cohesion variability range $10-20 \mathrm{kPa}$ (left) and 10-40 $\mathrm{kPa}$ (right), respectively 
Table 2. Results obtained analytically for zero and infinite thickness of random layer

\begin{tabular}{|c|c|c|c|c|c|c|c|c|c|}
\hline Range of cohesion var. [kPa] & \multicolumn{4}{|c|}{$10-20$} & \multicolumn{4}{c|}{$10-40$} \\
\hline \multicolumn{2}{|c|}{ Quantity } & \multicolumn{2}{|c|}{$P[\mathrm{kN}]$} & \multicolumn{2}{c|}{$h[\mathrm{~m}]$} & \multicolumn{2}{c|}{$P[\mathrm{kN}]$} & \multicolumn{2}{c|}{$h[\mathrm{~m}]$} \\
\hline \multicolumn{2}{|c|}{ Mean and stanard deviation } & $\mu_{P}$ & $\sigma_{P}$ & $\mu_{h}$ & $\sigma_{h}$ & $\mu_{P}$ & $\sigma_{P}$ & $\mu_{h}$ & $\sigma_{h}$ \\
\hline \multirow{2}{*}{$\begin{array}{c}\text { Random layer } \\
\text { thickness [m] }\end{array}$} & 0 & 76.1 & 0 & 0.645 & 0 & 117.4 & 0 & 0.592 & 0 \\
\cline { 2 - 11 } & $\infty$ & 82.11 & 15.76 & 0.707 & 0 & 136.8 & 47.41 & 0.707 & 0 \\
\hline
\end{tabular}
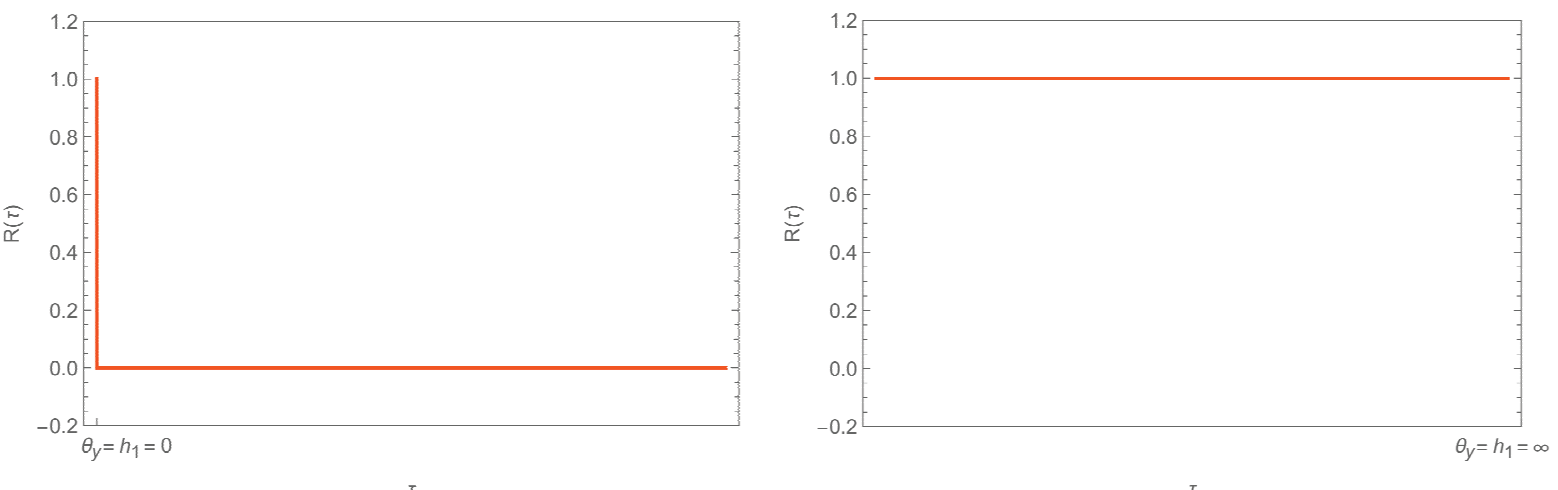

Fig. 10. Autocorrelation function for zero (left) and infinite (right) thickness of random layer

$$
\mu_{P}=b(2 \sqrt{2}+\sqrt{7}) \mu_{c}, \quad \sigma_{P}=b(2 \sqrt{2}+\sqrt{7}) \sigma_{c},
$$

where $\mu_{c}$ and $\sigma_{c}$ are mean and standard deviation for cohesion distribution.

Values of $P$ and $h$ obtained using the above formulas for the zero and infinity thickness and the cohesion described with uniform distribution in the ranges of $10-20 \mathrm{kPa}$ and $10-40 \mathrm{kPa}$ have been summarized in Table 2. The obtained mean values of assessment from both tables has been also presented in Fig. 9. As can be seen the mean value of assessment increases with the increasing thickness of the random layer.

It should be noted that for zero and infinite thickness of random layer the correlation functions differ from typical correlation function for finite non-zero thickness value presented in Fig. 2. The respective correlation function for this two extreme cases has been presented in Fig. 10.

\section{BEARING CAPACITY FOR GIVEN PROBABILITY OF FAILURE}

Probability of failure of foundation calculated in the framework of Monte-Carlo simulation is usually estimated using the formula:

$$
p_{F} \approx \frac{1}{N} \sum_{i=1}^{N} I\left[P_{i}<L\right]=\frac{n_{F}}{N},
$$

where $I[$.$] denotes indicator function, P_{i}$ is the bearing capacity in the realization, $L$ is the load (assumed here as deterministic) and $n_{F}$ is a number of failure cases. The problem can be inversed: based on formula (13) is also possible to calculate value of load such that the failure probability will be equal to given value, namely:

$$
L=x, \text { such that } \frac{1}{N} \sum_{i=1}^{N} I\left[P_{i}<x\right]=p_{F} .
$$

In order to satisfy (14) load value $x$ has to be greater than $p_{F}$ part of simulation results $P_{i}$

For all the cases presented in the previous section the appropriate values of load for assumed failure probability equal to 0.05 have been collected in Table 3 and presented graphically in Fig. 11. As can be seen load value corresponding to assumed probability of failure, contrary to the mean values of bearing capacity, decreases with increasing thickness of the random layer.

Table 3. The values of load corresponding to failure probability, equal to 0.05

\begin{tabular}{|c|c|c|c|}
\hline \multicolumn{2}{|c|}{ Range of cohesion $[\mathrm{kPa}]$} & $10-20$ & $10-40$ \\
\hline \multirow{4}{*}{$\begin{array}{l}\text { Random layer } \\
\text { thickness [m] }\end{array}$} & 0 & $76.10 \mathrm{kN}$ & $117.4 \mathrm{kN}$ \\
\cline { 2 - 4 } & 0.01 & $73.90 \mathrm{kN}$ & $110.3 \mathrm{kN}$ \\
\cline { 2 - 4 } & 0.05 & $70.84 \mathrm{kN}$ & $100.2 \mathrm{kN}$ \\
\cline { 2 - 4 } & 0.5 & $58.91 \mathrm{kN}$ & $64.46 \mathrm{kN}$ \\
\hline
\end{tabular}



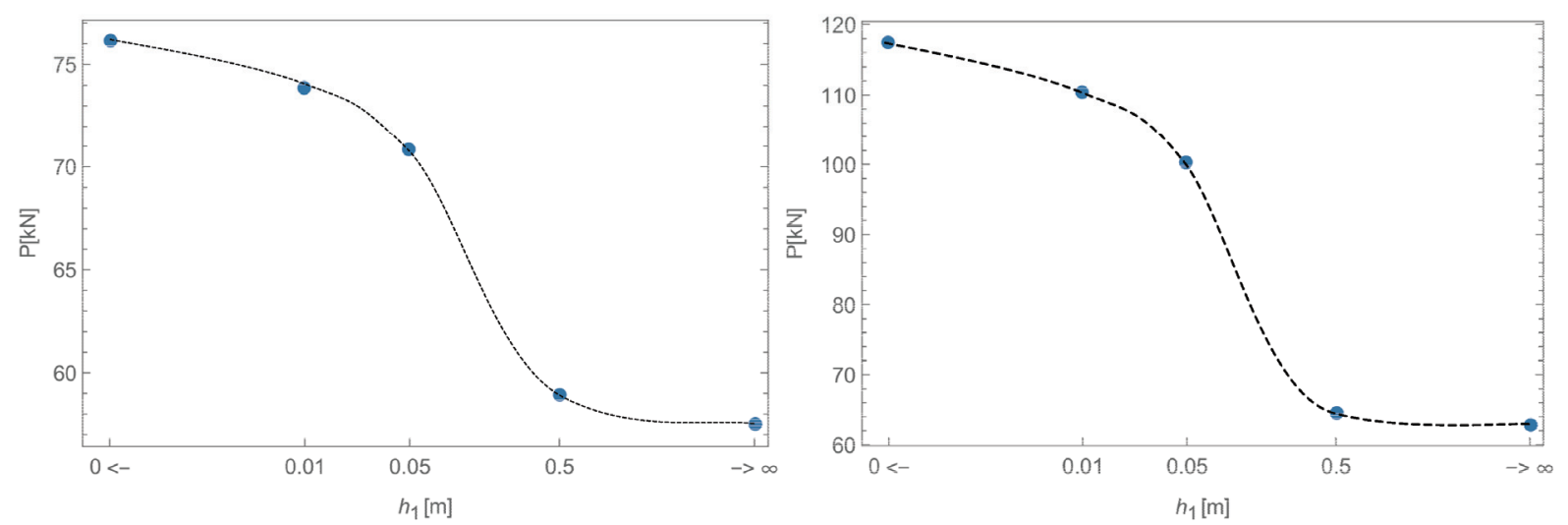

Fig. 11. The values of load corresponding to failure probability equal to 0.05 for cohesion variability range $10-20 \mathrm{kPa}$ (left) and $10-40 \mathrm{kPa}$ (right), respectively

\section{CONLUSIONS}

In the present paper concept of random layers has been proposed to obtain assessment of bearing capacity of strip footing. The presented approach combines random field theory in the form of random layers with classical upper bound limit analysis. The methodology is presented on the example of purely cohesive soil with the cohesion in the field described with uniform distribution and simple correlation structure. Formulation has been used within the framework of Monte-Carlo simulation. The results in the form of histograms and distribution for both bearing capacity of footing as well as optimal depth of failure mechanism has been presented. For the thickness of random layer tending to zero and infinity close form solutions has been derived. Based on the results of simulation and analytical solutions also the load, which corresponds to given probability of failure, has been calculated.

The following conclusions can be drawn from the study:

i) Simplification of the random field involving its conversion into set of horizontal random layers can lead to significant improvement in efficiency of the probabilistic modeling of soil. Since horizontal scale of fluctuation is usually several times greater than the vertical one, the result of probabilistic analysis performed with this simplification, in the case of the typical soil, should not be burdened with large errors.

ii) A classical upper bound limit analysis applied to random field can be a useful method for the probability analysis of geotechnical structures. By combining the method with the concept of random layers for which average value of the cohesion along dis- continuity lines can be efficiently calculated a very potent tool can be obtained.

iii) The mean value of bearing capacity of footing increases with increasing thickness of random layer. On the contrary, the load corresponding to assumed probability of failure decreases with increasing thickness of random layer. The latter tendency would be even more clear under the assumption of smaller (and therefore, from the point of view of design practice, more appropriate) probability of failure. This shows the importance of the application of random fields theory in geotechnics. In the classical approach, i.e. for homogeneous domain with random cohesion values a high mean value of bearing capacity is obtained, whereas value of load corresponding to given structural reliability is much smaller. Given the data on fluctuation scale, for the same probability of failure, usually much higher value of load can be obtained.

The current study is a preliminary one. The presented analysis are subject to at least two important limitations:

i) The results are presented for the field with the simplified correlation structure: the values of cohesion has been assigned to individual random layer independently. The resulting autocorrelation function decreases linearly. Usually some other correlation models are assumed. Generation of random layers with any other correlation structure is possible by extending the procedure using more detailed random field generation algorithm such as Local Average Subdivision (Fenton and Vanmarcke 1990).

ii) The uniform distribution of cohesion has been assumed which means that all values of cohesion within the variability range are assumed equally 
probable. Usually based on information obtained from geological studies some other types of distribution are assumed (e.g. Pieczyńska-Kozłowska et. al.). Type of distribution can have significant impact on failure probability. This impact should be examined and possibly some other, more appropriate distribution for cohesion should be selected. Overcoming of above limitation is subject of further work of the authors.

\section{REFERENCES}

Al-BitTar T., Soubra A.H., 2013, Bearing capacity of strip footings on spatially random soils using sparse polynomial chaos expansion, International Journal for Numerical and Analytical Methods in Geomechanics, 37 (13), 2039-2060.

FENTON G.A., GRIFFITHS D.V., 2003, Bearing-capacity prediction of spatially random $c \varphi$ soils, Canadian Geotechnical Journal, 40 (1), 54-65.
Fenton G.A., Vanmarcke E.H., 1990, Simulation of random fields via local average subdivision, Journal of Engineering Mechanics, 116(8), 1733-1749.

GrifFiths D.V., FENTON G.A., 2001, Bearing capacity of spatially random soil: the undrained clay Prandtl problem revisited, Geotechnique, 351-359.

Kasama Kiyonobu, Whittle A.J., Kouki Zen, 2012, Effect of spatial variability on the bearing capacity of cement-treated ground, Soils and Foundations 52.4, 600-619.

PieczyŃSKa-KozŁowsKa J.M., PuŁA W., Griffiths D.V., FENTON G.A., 2015, Influence of embedment, self-weight and anisotropy on bearing capacity reliability using the random finite element method, Computers and Geotechnics, 67, 229-238.

Uzielli M., Lacasse S., NAdim F., PhoOn K.K., 2006, Soil variability analysis for geotechnical practice, Characterization and Engineering Properties of Natural Soils, 3, 1653-1752.

Vessia G., Cherubini C., Pieczynska J., Pula W., 2009, Application of random finite element method to bearing capacity design of strip footing, Journal of Geoengineering, Vol. 4, No. 3.

VANMARCKe E., Random Fields: Analysis and Synthesis, MIT Press, 1983 